\title{
Drawing Planar Graphs with Circular Arcs*
}

\author{
C. C. Cheng, ${ }^{1}$ C. A. Duncan, ${ }^{2}$ M. T. Goodrich, ${ }^{3}$ and S. G. Kobourov ${ }^{4}$ \\ ${ }^{1}$ Mathematical Sciences Department, Johns Hopkins University, \\ Baltimore, MD 21218, USA \\ ${ }^{2}$ Department of Computer Science, University of Miami, \\ Coral Gables, FL 33124, USA \\ ${ }^{3}$ Department of Computer Science, Johns Hopkins University, \\ Baltimore, MD 21218, USA \\ ${ }^{4}$ Department of Computer Science, University of Arizona, \\ Tucson, AZ 85721, USA
}

\begin{abstract}
In this paper we address the problem of drawing planar graphs with circular arcs while maintaining good angular resolution and small drawing area. We present a lower bound on the area of drawings in which edges are drawn using exactly one circular arc. We also give an algorithm for drawing $n$-vertex planar graphs such that the edges are sequences of two continuous circular arcs. The algorithm runs in $O(n)$ time and embeds the graph on the $O(n) \times O(n)$ grid, while maintaining $\Theta(1 / d(v))$ angular resolution, where $d(v)$ is the degree of vertex $v$. Since in this case we use circular arcs of infinite radius, this is also the first algorithm that simultaneously achieves good angular resolution, small area, and at most one bend per edge using straight-line segments. Finally, we show how to create drawings in which edges are smooth $C^{1}$-continuous curves, represented by a sequence of at most three circular arcs.
\end{abstract}

\section{Introduction}

The study of methods for rendering planar graphs is central in the graph drawing literature. In planar graph drawings, vertices are represented by distinct points in the plane and edges are drawn as continuous curves that do not cross one another [1]. An important characteristic of a graph drawing is its readability, and some of the essential qualities

* A preliminary version of this paper appeared in the Proceedings of the 7th Annual Symposium on Graph Drawing, 1999. The first author was partially supported by ONR Grant N00014-96-1-0829, the other three authors were partially supported by NSF Grant CCR-9732300 and ARO Grant DAAH04-96-1-0013. 
that determine readability include the following:

1. Edge smoothness: edges should be drawn with "smooth" curves. Ideally, we prefer straight-line segments. If some other considerations prevent the use of straight lines, then edges should be drawn as simple smooth low-degree curves or polylines with few bends.

2. Area: vertices and bend points should be placed at integer grid points in as small a box as possible. Ideally, vertices and bend points should be placed on an $O(n) \times$ $O(n)$ grid, where $n$ is the number of vertices in the graph.

3. Angular resolution: for each pair $s$ and $t$ of curves representing two consecutive edges incident on a vertex $v$, the angle between the tangent of $s$ at $v$ and the tangent of $t$ at $v$ should be large. Ideally, we would like the measure of each such angle to be $\Theta(1 / d(v))$, where $d(v)$ denotes the degree of $v$.

Thus, we are interested in a study of methods for drawing planar graphs with smooth edges, small area, and ideal angular resolution. The particular emphasis in this paper is to consider methods for drawing edges with polylines such that each piece of the polyline is drawn with a circular arc. This is a strict generalization of the usual piecewise-linear polylines [8], [11], since a straight-line segment can be viewed as an arc of a circle of infinite radius. In this paper we address the following questions: What area is achievable for drawings with good angular resolution that use single circle arcs for edges? What area is achievable for drawings that use at most two circular arcs per edge and have good angular resolution? What is the fewest number of circular arcs needed to achieve $O(n) \times O(n)$ area, good angular resolution, and $C^{1}$-continuity for edges?

\subsection{Prior Related Work}

There is a rich body of knowledge that has been developed for drawing planar graphs. Early work by Wagner [15], Fáry [4], and Tutte [14] focused on drawings of planar graphs using straight-line edges, without much attention paid to other aesthetic or complexity issues. Indeed, the drawings produced using these early techniques can in many cases require exponential area. Later de Fraysseix et al. [3] and then Schnyder [13] showed that one can draw a planar graph with straight-line edges and vertices placed at grid points in an $O(n) \times O(n)$ integer grid. Still, the drawings produced from these algorithms have a weakness, which is not as prevalent in the algorithms based on Tutte's approach: namely, the area-efficient straight-line drawings can produce very small angles between consecutive edges incident upon the same vertex (poor angular resolution). In fact, it has been proven by Malitz and Papakostas [12] that there exist graphs that always require exponential area for straight-line embeddings maintaining good angular resolution.

The problem of drawing planar graphs with good angular resolution was addressed by Formann et al. [5], Garg and Tamassia [6], and Kant [9]-[11], who showed that one could in fact simultaneously achieve $O(n) \times O(n)$ area and an angular resolution of $\Theta(1 / d(v))$ for each vertex $v$, by drawing a planar graph using piecewise-linear polylines with at most three bends each. Gutwenger and Mutzel [8] improved the constant factors for such drawings, establishing that one could draw an $n$-vertex planar graph in a $(2 n-5) \times(3 n / 2-7 / 2)$ grid with at least $2 / d_{\max }$ angular resolution using piecewise- 
linear polylines with at most three bends each, where $d_{\max }$ is the maximum degree of the graph. Goodrich and Wagner [7] showed that one could in fact achieve $O(n) \times O(n)$ area with an angular resolution of $\Theta(1 / d(v))$ for each vertex $v$, using piecewise-linear polylines with only two bends each. They also showed that one could achieve the same area and angular resolution bounds using smooth degree-3 (Bézier) curves.

\subsection{Our Results}

In this paper we provide answers to the questions posed above. Specifically, we show the following:

- There exists an $n$-vertex planar graph $G$ that requires area exponential in $n$ for any drawing of $G$ that uses single circle arcs for edges and has good angular resolution.

- We can draw an $n$-vertex planar graph $G$ in an $O(n) \times O(n)$ grid with angular resolution $\Theta(1 / d(v))$ for each vertex $v$ in $G$ using at most two circular arcs per edge. In fact, in this case we use circular arcs of infinite radius so that the polylines are piecewise linear with at most one bend each, while still maintaining good angular resolution and $O(n) \times O(n)$ area.

- We can draw an $n$-vertex planar graph $G$ in an $O(n) \times O(n)$ grid with angular resolution $\Theta(1 / d(v))$ for each vertex $v$ in $G$ using $C^{1}$-continuous curves that consist of at most three circular arcs.

Our lower-bound proof is based on a nontrivial analysis of a circular-arc drawing of the well-known nested-triangles graph. Our algorithm is based on a careful modification of the incremental approach to planar graph drawing utilized by de Fraysseix et al. [3] similar to the approach used by Goodrich and Wagner [7]. We describe the main ideas behind these results in the sections that follow, beginning our discussion with the algorithm.

\section{Algorithm}

We now describe an efficient algorithm, OneBend, to embed any planar graph on an $O(n) \times O(n)$ grid while maintaining good angular resolution, $\Theta(1 / d(v))$, for each vertex $v$, and using at most one bend per edge. Following the methods of de Fraysseix et al. [3] and Kant [11], we insert vertices sequentially by their canonical ordering, generating subgraphs $G_{1}, G_{2}, \ldots, G_{n}$ in the process. Recall that in the canonical order, vertices are labeled $v_{1}, v_{2}, \ldots, v_{n}$ and graph $G_{i}$ is defined to be the subgraph induced on the vertices $v_{1}, v_{2}, \ldots, v_{i}$. Graph $G_{i}$ is 2-connected and its external face is a cycle $C_{i}$. Furthermore, in graph $G_{i+1}$, the new vertex, $v_{i+1}$ has all of its neighbors on the external face of $C_{i}$.

In the manner of Goodrich and Wagner [7], we use a box around each vertex of size proportional to its degree but guarantee that each edge drawn contains at most one bend rather than the previous best known method using two. To generate a subgraph $G_{k+1}$ from $G_{k}$ by inserting a vertex $v_{k+1}$ and its associated box, we need to perform a few operations and maintain a few sets. Let $w_{1}=v_{1}, w_{2}, \ldots, w_{m}=v_{2}$ be the vertices of the exterior face $C_{k}$ of $G_{k}$ in order. For a particular subgraph $G_{k}$ and vertex $v_{k+1}$, we refer to $w_{l}$ and $w_{r}$ as the leftmost and rightmost neighbors of $v_{k+1}$ on $C_{k}$; see Fig. 1 . 


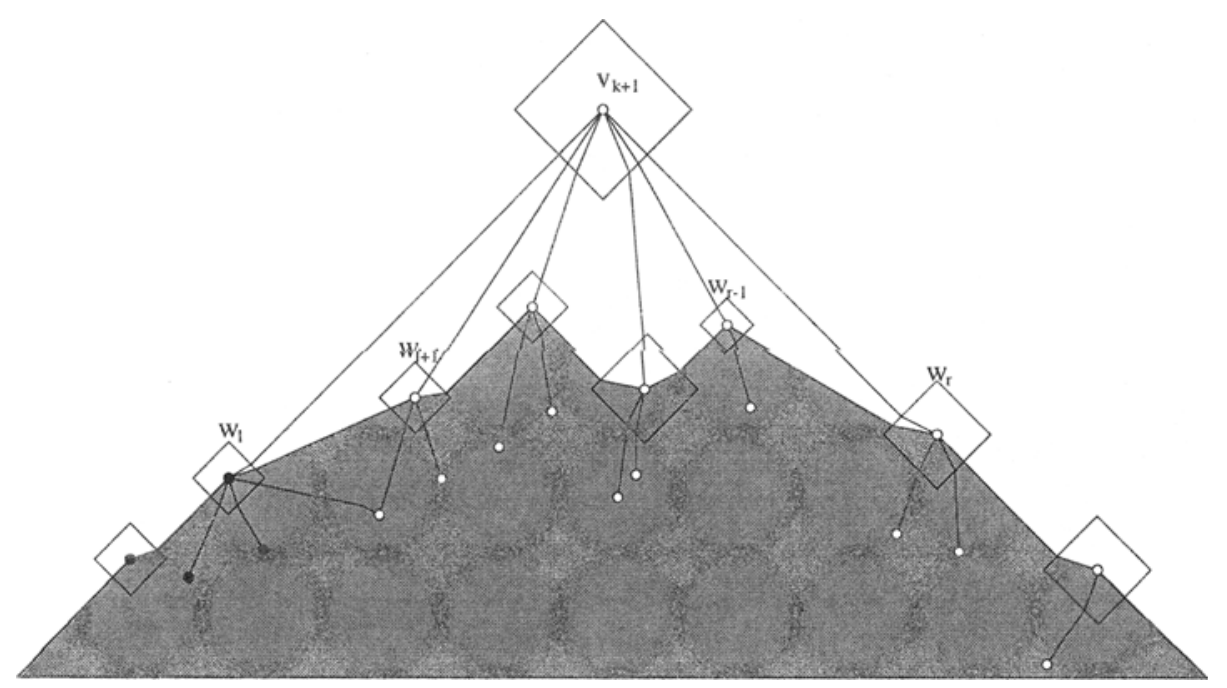

Fig. 1. Graph $G_{k+1}$ after inserting $v_{k+1}$. The shaded part is $G_{k}$ and all unfilled vertices are part of the shifting set $M_{k+1}\left(v_{k+1}\right)$.

\subsection{Vertex Joint Box}

We associate with every vertex $v \in V$ a joint box centered around $v$, rotated $45^{\circ}$, and having width and height $4 d(v)+4$ units, see Fig. 2 . For notational convenience, if $v$ is clear from the context, then we use $d$ to denote the degree, $d(v)$, of $v$. Thus, if $v$ is located at position $(i, j)$, the four corners of the box are $(i \pm 2 d+2, i)$ and $(i, j \pm 2 d+2)$. We break the box into two types of alternating regions, free regions and port regions. For each

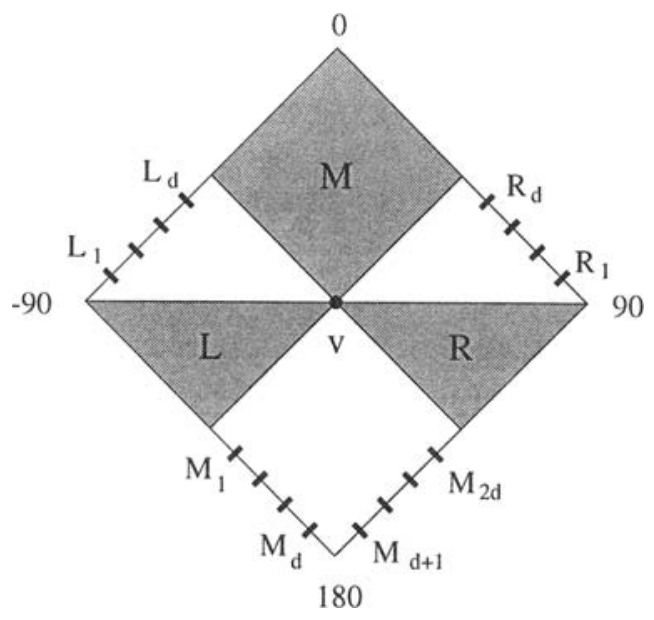

Fig. 2. The joint box for a vertex, $v$, in the drawing. Notice the shaded regions highlighting the three free regions and the presence and location of ports inside the port regions. 


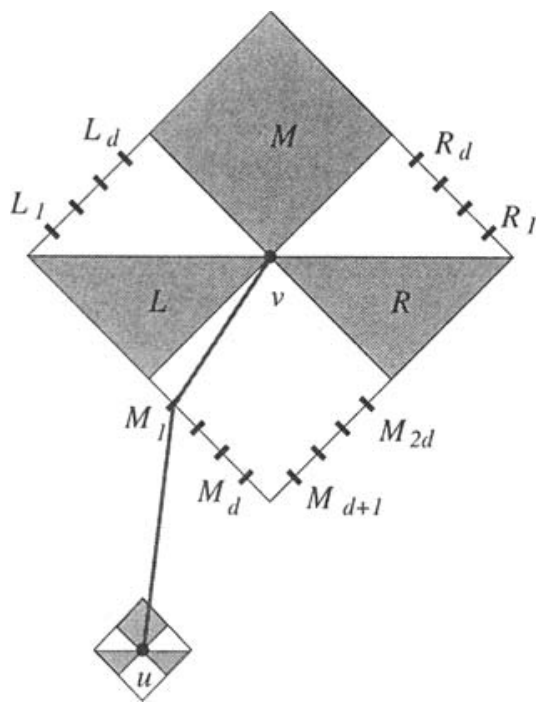

Fig. 3. The edge from $u$ to $v$ has two edge segments. The free edge segment connects $u$ to $M_{1}$. The port edge segment connects $v$ to $M_{1}$.

free region there is at most one edge passing through it to $v$. Each port region consists of a collection of $d$ ports and every edge inside the port region passes through a unique port.

We define and name the free regions using angular coordinates clockwise around $v$ :

- Free region $M$ lies between $-45^{\circ}$ and $45^{\circ}$.

- Free region $R$ lies between $90^{\circ}$ and $135^{\circ}$.

- Free region $L$ lies between $-135^{\circ}$ and $-90^{\circ}$.

In between each of these regions are the port regions. For reference, we label the ports between $L$ and $M$ upward as $L_{1}, \ldots, L_{d}$ and similarly between $R$ and $M$. The ports between $L$ and $R$ are labeled $M_{1}, M_{2}, \ldots, M_{2 d}$ in counterclockwise order.

The algorithm draws each edge in $E$ by "routing" it through a port in the joint box of one of the two endpoints. Each edge consists of two connected edge segments. The first edge segment, the port edge segment, connects a vertex with one of the ports of its joint box. The second edge segment, the free edge segment, connects a vertex to one of its neighbor's ports. For example, for an edge $e=(u, v)$, if we route $e$ through port $M_{1}$ in $v$ 's joint box, we would draw two line segments, see Fig. 3 . The free edge segment would pass from $u$ to $M_{1}$ and the port edge segment would pass from $v$ to $M_{1}$. This method of construction enables us to guarantee that there is at most one bend per edge and that the free edge segments always pass through free regions.

\subsection{The Invariants}

In order to construct our embedding incrementally, we maintain invariants similar to those of de Fraysseix et al. [3] and Goodrich and Wagner [7] with two differences, a 
slight change in invariant three and a new invariant four:

1. The vertices and the ports of the joint boxes have integer coordinates.

2. Let $w_{1}=v_{1}, w_{2}, \ldots, w_{m}=v_{2}$ be the vertices of the exterior face $C_{k}$ of $G_{k}$ in order and let $x\left(w_{i}\right)$ be the $x$-coordinate of vertex $w_{i}$. Then $x\left(w_{1}\right)<x\left(w_{2}\right)<$ $\cdots<x\left(w_{m}\right)$.

3. For $0<i<m$ the free edge segment of the edge $\left(w_{i}, w_{i+1}\right)$ has slope \pm 1 .

4. For every vertex $v$ there is at most one (free) edge segment crossing each of its free regions, see Fig. 2. All other edge segments are port edge segments.

Notice that if invariant four holds for the embedding $G_{k}$, by the definition of the joint box and location of the port regions, $G_{k}$ has angular resolution no worse than $\Theta(1 / d(v))$, for each vertex $v$.

\subsection{The Shifting Set}

During each insertion, we must create space for the vertex joint box to "see" its leftmost and rightmost neighbors without the box touching any of the neighbors along the face in between. To do this, we need to shift the vertices along the external face by a certain amount. However, in order for the invariants and planarity to be guaranteed other vertices must also be shifted at the same time. As in de Fraysseix et al. [3] and Goodrich and Wagner [7], we define the shifting set for a vertex $w_{i}$ on the external face of $G_{k}$ as $M_{k}\left(w_{i}\right)$. For any graph $G_{k}$, we define $M_{k}\left(w_{i}\right) \subseteq V$ such that the following conditions hold:

1. $w_{j} \in M_{k}\left(w_{i}\right)$ if and only if $j \geq i$.

2. $M_{k}\left(w_{1}\right) \supset M_{k}\left(w_{2}\right) \supset \cdots \supset M_{k}\left(w_{m}\right)$.

3. For any nonnegative number $\delta_{1}, \delta_{2}, \ldots, \delta_{m}$ if we sequentially translate all vertices in $M_{k}\left(w_{i}\right)$ with distance $\delta_{i}$ to the right $(i=1,2, \ldots, m)$, then the embedding of $G_{k}$ remains planar. $^{1}$

Recall that for a vertex $v=v_{k+1}, w_{l}$ and $w_{r}$ are the leftmost and rightmost neighbors of $v$ on $C_{k}$. Starting with the initial shifting set at $k=3$, we construct $M_{k+1}\left(w_{i}\right)$ recursively as follows:

- $M_{k+1}\left(w_{i}\right)=M_{k}\left(w_{i}\right) \cup v_{k+1}$, for $i \leq l$.

- $M_{k+1}\left(v_{k+1}\right)=M_{k}\left(w_{l+1}\right) \cup v_{k+1}$.

- $M_{k+1}\left(w_{j}\right)=M_{k}\left(w_{j}\right)$, for $j \geq r$.

This construction allows us to guarantee that the above three conditions of the shifting sets are maintained. Intuitively, after a vertex $w_{i}$ is removed from the external face by another vertex $v_{k+1}$, it always shifts exactly with $v_{k+1}$. During any shift, vertices can only get farther apart in the $x$-direction. Note that in our algorithm, when a vertex is shifted, its joint box is also shifted, that is, the ports move as well.

\footnotetext{
${ }^{1}$ Note that many vertices will move several times; e.g. all points in $M_{k}\left(w_{i}\right) \backslash M_{k}\left(w_{i+1}\right)$ will be translated by $\delta_{1}+\delta_{2}+\cdots+\delta_{i}$.
} 


\subsection{The Construction}

We first show how algorithm OneBend iteratively constructs the sequence of graphs $G_{1}, G_{2}, \ldots, G_{n}$. It is trivial to construct the initial cases of $G_{1}, G_{2}$, and $G_{3}$, i.e., inserting the first three vertices. Suppose we have embedded $G_{k}$ with exterior face $C_{k}$. Let $C_{k}=$ $\left(v_{1}=w_{1}, w_{2}, \ldots, w_{m}=v_{2}\right)$ be the exterior face of $G_{k}$. To construct $G_{k+1}$, let $v=v_{k+1}$ be the next vertex in the canonical ordering and recall that $w_{l}$ and $w_{r}$ are, respectively, the leftmost and rightmost neighbors of $v$ on the face $C_{k}$. Let $d, d_{l}, d_{r}$ be the respective degrees of $v, w_{l}$, and $w_{r}$. Let $p_{l}$ be the first unused $R_{i}$ port in $w_{l}$ 's joint box. Similarly, let $p_{r}$ be the first unused $L_{i}$ port in $w_{r}$ 's joint box. Recall since each port region has at least $d$ ports available there is always an unused port.

We insert $v$ by shifting all vertices in the shifting set $M_{k}\left(w_{l+1}\right)$ by $2 d+2$ positions to the right. Additionally we shift all vertices in $M_{k}\left(w_{r}\right)$ by an additional $2 d+2$ positions to the right. This implies all vertices in $M_{k}\left(w_{r}\right)$ actually move $4 d+4$ positions. Finally, we place $v$ at the intersection of lines $l$ and $r$ where $l$ (respectively $r$ ) is the line through $p_{l}$ (respectively $p_{r}$ ) with slope +1 (respectively -1 ). We route the edges between $v$ and $w_{l}$ through $p_{l}$ and do the same for $w_{r}$. To maintain invariants one and three, notice that if the intersection point has integer coordinates these two invariants hold. Otherwise, by shifting $M_{k}\left(w_{r}\right)$ one additional unit, we guarantee that the intersection point has integer coordinates.

To complete the insertion and the algorithm, we need to draw the edges between $v$ and $w_{i}$, where $l<i<r$. Let $w_{j}$ be the rightmost vertex with an $x$-coordinate less than $v$. We route the edges from $v$ to vertices $w_{j}$ where $l<i \leq j$ through consecutive increasing ports from $M_{1}$ in $v$ 's joint box. Similarly, we route the edges from $v$ to vertices $w_{i}$, where $r>i>j$ through consecutive decreasing ports from $M_{2 d}$ in $v$ 's joint box.

Lemma 1. After shifting, any free edge segment in the free region remains in the free region.

Proof. We first look at free edge segments in the $M$ regions. Notice that these segments are created by a vertex $v$ dominating another vertex $w$. In this case, $w$ joins $v$ 's shifting set and is only shifted whenever $v$ is shifted. Therefore, the slope remains constant and the free edge segment remains within $M$.

All other free edge segments lie inside $L$ and $R$ free regions. Without loss of generality, we examine the case when a free edge segment lies in the $L$ region. This implies that the slope of the line segment is between 0 and +1 . Since shifting only moves vertices farther apart in the $x$-direction, the slope can get closer to 0 but it will always remain positive. Thus the free edge segment will always remain in the $L$ free region. The argument is similar for free edge segments in the $R$ free regions.

Lemma 2. After insertion, every free edge segment passes through a free region which contains no other segment.

Proof. After inserting a vertex $v_{k+1}$, there are two types of edges added, the edges between $v_{k+1}$ and the outside neighbors, $w_{l}$ and $w_{r}$, and between $v_{k+1}$ and $w_{i}$ where 
$l<i<r$. In both cases, each edge is routed through a port creating one free edge segment and one port edge segment. By construction, a free edge segment of the first type by construction has slope either +1 or -1 and so it lies inside $v_{k+1}$ 's joint box free region $L$ or $R$. Since $v_{k+1}$ is a new vertex, there are no other segments inside these two free regions.

A free edge segment of the second type intersects the $M$ region of $w_{i}$ 's joint box. Since this can happen at most once, as the vertex is now no longer on an external face, there can be no other free edge segment inside this free region. To see that the segment actually intersects the $M$ free region, notice that $w_{i}$ is bound between $w_{l}$ and $w_{r}$ and is also added into a port on the proper side of $v_{k+1}$, i.e., the left or right side. Also, by construction $w_{i}$ is below the lowest port of $v_{k+1}$ 's joint box. This implies that the slope is bound either above +1 or below -1 and, therefore, the free edge segment lies inside the $w_{i}$ 's $M$ region.

Lemma 3. If invariants 1-4 hold for $G_{k}$, then they also hold for $G_{k+1}$.

Proof. By the nature of the shifting set, invariants one and two hold (see [7]). Note that because shifting a vertex involves shifting the entire joint box simultaneously, after every shift operation all port edge segments have unchanged slope. Also, after the two shifting operations, all free edge segments on the exterior face have unchanged, albeit \pm 1 , slope, except possibly the free edge segments $\left(w_{l}, w_{l+1}\right)$ and $\left(w_{r-1}, w_{r}\right)$. However, after insertion, these free edge segments are no longer on the exterior face and are instead replaced by two free edge segments between $\left(w_{l}, v\right)$ and $\left(v, w_{r}\right)$ with slope \pm 1 . Therefore, invariant three holds.

By Lemmas 1 and 2 and the fact that port edge segments never change slope, we see that invariant four also holds since all edges routed in algorithm OneBend created a port segment and a free edge segment.

Theorem 1. Given a planar graph $G$, algorithm OneBend produces in $O(n)$ time a planar embedding on the $30 n \times 15 n$ grid with angular resolution $\Theta(1 / d(v))$ and using any of the following types of edges: polylines with one bend, or two circular arcs with $C^{0}$-continuity and one knot, or three circular arcs with $C^{1}$-continuity.

Proof. The original algorithm as stated produces polylines with one bend per edge. This by definition can also be represented by two circular arcs, straight lines, which have a discontinuity at the bend, or knot. Since the points are embedded on the grid, the bends may also be replaced by circular arcs of a relatively small size to ensure $C^{1}$-continuity as well.

It has been shown by Chrobak and Payne [2] how to implement the algorithm of de Fraysseix et al. [3] in linear time. Their approach can be easily extended to our algorithm.

It remains to show that the drawings produced by algorithm oneBend fit on the $30 n \times 15 n$ grid. Every time we insert a vertex $v_{k}$, we increase the grid size by $4 d\left(v_{k}\right)+5$ units. Summing over all the degrees of the vertices we get $\sum_{v \in V} 4 d(v)+5=4(6 n-$ 12) $+5 n<30 n$. The final drawing fits inside an isosceles triangle with sides of slope $0,+1,-1$. The width of the base is $30 n$ and so the height is less than $15 n$. 


\section{Drawing with Circular Ares}

Malitz and Papakostas [12] showed that some planar graphs, drawn with straight lines in the $O(n) \times O(n)$ grid, must have small angles. More specifically, they found a class of planar graphs, $\mathcal{H}$, whose straight-line planar drawings require exponential area if the angular resolution is good. Suppose we relax the condition that each edge in a graph be drawn with a straight-line segment so that each edge is drawn with a circular arc (where a straight-line segment is considered an arc from a circle of radius infinity). Can we draw the graphs in $\mathcal{H}$ with angular resolution $\alpha>0$ in an $O(n) \times O(n)$ grid? Surprisingly, as long as $\alpha$ is a constant, the answer is no.

Let $\mathcal{H}=\left\{H_{n}, n \geq 1\right\}$ and $H_{1}$ be a cycle on three vertices $P_{1}, Q_{1}$, and $R_{1}$. For $n \geq 2$, the graph $H_{n}$ is constructed from $H_{n-1}$ by adding a cycle on three new vertices $P_{n}, Q_{n}, R_{n}$, and edges $\left(P_{n}, P_{n-1}\right),\left(Q_{n}, Q_{n-1}\right),\left(R_{n}, R_{n-1}\right)$ and $\left(P_{n}, Q_{n-1}\right),\left(Q_{n}, R_{n-1}\right)$, $\left(R_{n}, P_{n-1}\right)$, as shown in Fig. 4 . It is easy to check that the graph is planar, triconnected, and, thus, has a unique embedding. We show that for any planar, circular-arc drawing of $H_{n}$ with angular resolution $\alpha>0$, there exists a constant $c_{\alpha}>1$ such that the area of the drawing is $\Omega\left(c_{\alpha}^{n}\right)$.

Let $\Gamma_{n}$ be a planar circular-arc drawing of $H_{n}$ with angular resolution $0<\alpha \leq \pi / 3$. If $(u, v)$ is an edge in $H_{n}$, then we refer to the arc that represents $(u, v)$ in $\Gamma_{n}$ as $\widehat{u v}$, and the line segment that connects $u$ and $v$ as $\overline{u v}$. (Sometimes $u$ or $v$ may not be a vertex of $H_{n}$ but a point on some arc of $\Gamma_{n}$. In this case, $\widehat{u v}$ refers to the portion of the arc that starts at $u$ and ends at $v$.) If $\mathcal{S}$ is a set of arcs in $\Gamma_{n}$ that bounds a region, then we let $\operatorname{Area}(\mathcal{S})$ be its area.

We next define regions $\mathcal{S}_{1}, \mathcal{S}_{2}$, and $\mathcal{S}_{3}$ as follows:

$$
\begin{aligned}
& \mathcal{S}_{1}=\left\{\widehat{P_{n-1} Q_{n-1}}, \widehat{Q_{n-1} P_{n}}, \widehat{P_{n} P_{n-1}}\right\} \\
& \mathcal{S}_{2}=\left\{\widehat{Q_{n-1} R_{n-1}}, \widehat{R_{n-1} Q_{n}}, \widehat{Q_{n} Q_{n-1}}\right\}, \\
& \mathcal{S}_{3}=\left\{\widehat{R_{n-1} P_{n-1}}, \widehat{P_{n-1} R_{n}}, \widehat{R_{n} R_{n-1}}\right\} .
\end{aligned}
$$

We show in the next two lemmas that the region enclosed by the three $\operatorname{arcs}$ in $\mathcal{S}_{1}$ cannot
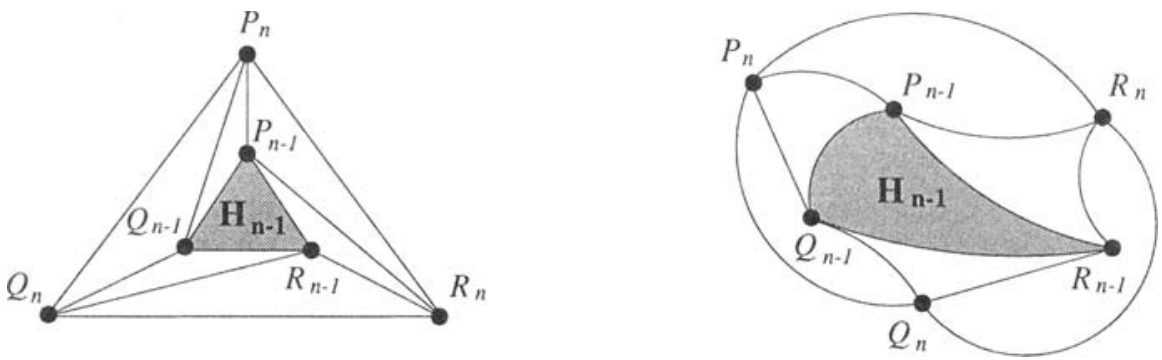

Fig. 4. The graph $H_{n}$ is constructed from $H_{n-1}$ by adding vertices $P_{n}, Q_{n}$, and $R_{n}$ along with the edges shown above. The figure on the right shows $H_{n}$ drawn with circular arcs. 

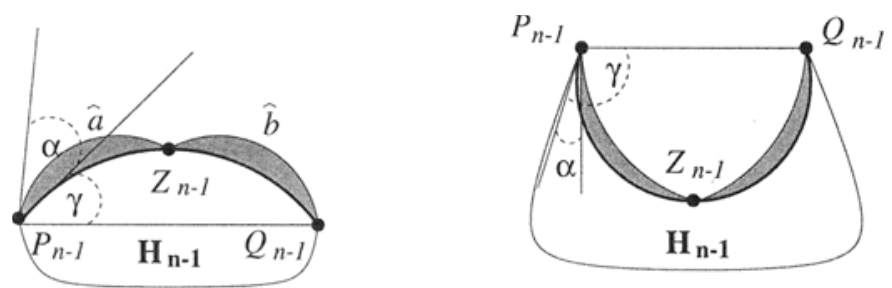

Fig. 5. Arcs $\widehat{a}$ and $\widehat{b}$ pass through $P_{n-1}, Z_{n-1}$, and $Q_{n-1}, Z_{n-1}$, respectively. Their tangents form an angle $\alpha$ with the tangents of $\widehat{P_{n-1} Z_{n-1}}$ and $\widehat{Q_{n-1} Z_{n-1}}$. The shape of the region bounded by $\widehat{a}$ and $\widehat{P_{n-1} Z_{n-1}}$ depends on the concavity/convexity of $P_{n-1} Q_{n-1}$ and $\alpha$.

be arbitrarily small. If all the arcs in $H_{n}$ are straight lines, this fact is easy to prove. However, for circular-arc drawings, we need to take into account that the arcs can have different curvatures. The requirement that the tangents of two incident arcs must form at least an angle $\alpha>0$ will allow us to show that $A$ rea $\left(\mathcal{S}_{1}\right)$ is proportional to $\left|\overline{P_{n-1} Q_{n-1}}\right|^{2}$. Similarly, the areas of the regions enclosed by the arcs in $\mathcal{S}_{2}$ and $\mathcal{S}_{3}$ cannot be arbitrarily small.

Let $Z_{n-1}$ be the midpoint of $\widehat{P_{n-1} Q_{n-1}}$. Consider the two circular arcs that pass through $P_{n-1}$ and $Z_{n-1}$ such that the tangents of the arcs form an angle $\alpha$ with $\widehat{P_{n-1} Q_{n-1}}$. Let $\widehat{a}$ be the arc that lies on the outside face of $H_{n-1}$. Let $\widehat{b}$ be the corresponding arc that passes through $Q_{n-1}$ and $Z_{n-1}$, see Fig. 5 .

Lemma 4. $\operatorname{Area}\left(\mathcal{S}_{1}\right) \geq \operatorname{Area}\left(\left\{\widehat{a}, \widehat{P_{n-1} Z_{n-1}}\right\}\right)$.

Proof. Let $l$ be the perpendicular bisector of $\overline{P_{n-1} Q_{n-1}}$. Without loss of generality, assume that $P_{n}$ lies on $l$ or on the same side of $l$ as $Q_{n-1}$. Notice that if $P_{n} \neq Z_{n-1}$, $\widehat{P_{n-1} P_{n}}$ is always above $\widehat{a}$ except at its endpoint, $P_{n-1}$. Otherwise, the angular resolution of $\Gamma_{n}$ is violated or $P_{n}$ lies below $\widehat{a}$ and hence on the wrong side of $l$. Furthermore, $\widehat{Q_{n-1} P_{n}}$ cannot intersect $\widehat{a}$, except possibly at $Z_{n-1}$. If it does, it crosses $l$ and has to intersect $\widehat{P_{n-1} P_{n}}$ as well, contradicting the assumption that $\Gamma_{n}$ is a planar drawing. Thus, both $\widehat{P}_{n-1} P_{n}$ and $\widehat{Q}+1_{n-1}$ do not cross $\widehat{a} ; \widehat{a}$ must lie in the region enclosed by $\mathcal{S}_{1}$. By symmetry, if $P_{n}$ lies on the same side of $l$ as $P_{n-1}$, then $\widehat{b}$ must lie in the region enclosed by $\mathcal{S}_{1}$. Our result follows.

Lemma 5. There exist positive constants $k_{\alpha}$ and $k_{\alpha}^{\prime}$ such that

(i) $\operatorname{Area}\left(\left\{\widehat{a}, \widehat{P_{n-1} Z_{n-1}}\right\}\right) \geq k_{\alpha}\left|\overline{P_{n-1} Q_{n-1}}\right|^{2}$ and

(ii) $\operatorname{Area}\left(\left\{\hat{a}, \widehat{P_{n-1} Z_{n-1}}\right\}\right) \geq k_{\alpha}^{\prime} \operatorname{Area}\left(\left\{P_{n-1} Q_{n-1}, \overline{P_{n-1} Q_{n-1}}\right\}\right)$.

Proof. Without loss of generality, we assume that $P_{n-1}$ is on the origin and $Q_{n-1}$ is on the positive $x$-axis. The area enclosed by $\widehat{a}$ and $\widehat{P_{n-1} Z_{n-1}}$ depends on the convexity or concavity of $\widehat{P_{n-1} Q_{n-1}}$. when $\widehat{P_{n-1} Q_{n-1}}$ is convex and when $\widehat{P_{n-1} Q_{n-1}}$ is concave.

We assume $P_{n-1} Q_{n-1}$ is convex. Let $\gamma<\pi$ be the angle formed between $\overline{P_{n-1} Q_{n-1}}$ and the tangents of $\widehat{P_{n-1} Q_{n-1}}$ at $P_{n-1}$ and $Q_{n-1}$. When $\gamma \leq 2 \alpha, \widehat{a}$ is concave. Let 
$f(\theta)=(\theta-\sin \theta) /(1-\cos \theta)$. It is easy to verify that

- $\operatorname{Area}\left(\left\{\hat{a}, \overline{P_{n-1} Z_{n-1}}\right\}\right)=\left|\overline{P_{n-1} Z_{n-1}}\right|^{2} f(2 \alpha-\gamma) / 4$,

- $\operatorname{Area}\left(\left\{\overline{P_{n-1} Z_{n-1}}, \overline{P_{n-1} Z_{n-1}}\right\}\right)=\left|\overline{P_{n-1} Z_{n-1}}\right|^{2} f(\gamma) / 4$,

- $\operatorname{Area}\left(\left\{\widehat{a}, \widehat{P_{n-1} Z_{n-1}}\right\}\right)=\operatorname{Area}\left(\left\{\widehat{a}, \overline{P_{n-1} Z_{n-1}}\right\}\right)+\operatorname{Area}\left(\left\{\overline{P_{n-1} Z_{n-1}}, \widehat{P_{n-1} Z_{n-1}}\right\}\right)$.

Thus,

$$
\begin{aligned}
\operatorname{Area}\left(\left\{\widehat{a}, \widehat{\left.\left.P_{n-1} Z_{n-1}\right\}\right)}\right.\right. & =\left|\overline{P_{n-1} Z_{n-1}}\right|^{2} \frac{f(2 \alpha-\gamma)+f(\gamma)}{4} \\
& \geq\left|\overline{P_{n-1} Q_{n-1}}\right|^{2} \frac{f(\alpha)}{16 \cos ^{2}(\gamma / 2)} \\
& \geq\left|\overline{P_{n-1} Q_{n-1}}\right|^{2} \frac{f(\alpha)}{16},
\end{aligned}
$$

where (1) follows from the fact that $f(\theta)$ is monotonically increasing when $0<$ $\theta<\pi$ and $0 \leq \gamma \leq 2 \alpha$. Furthermore, since $\gamma \leq 2 \alpha$, the largest possible value of Area $\left(\left\{\widehat{P_{n-1} Q_{n-1}}, \overline{P_{n-1} Q_{n-1}}\right\}\right)$ is $\left|\widetilde{P_{n-1} Q_{n-1}}\right|^{2} f(4 \pi / 3) / 4$. Hence,

$$
\operatorname{Area}\left(\left\{\widehat{a}, \widehat{P_{n-1} Z_{n-1}}\right\}\right) \geq \operatorname{Area}\left(\left\{\widehat{P_{n-1} Q_{n-1}}\right\}\right) \frac{f(\alpha)}{4 f(4 \pi / 3)} .
$$

If $\gamma>2 \alpha$, then $\widehat{a}$ is convex. Again, it is easy to verify that

- $\operatorname{Area}\left(\left\{\hat{a}, \overline{P_{n-1} Z_{n-1}}\right\}\right)=\left|\overline{P_{n-1} Z_{n-1}}\right|^{2} f(\gamma-2 \alpha) / 4$.

- $\operatorname{Area}\left(\left\{\hat{a}, \widehat{P_{n-1} Z_{n-1}}\right\}\right)=\operatorname{Area}\left(\left\{\overline{P_{n-1} Z_{n-1}}, \widehat{P_{n-1} Z_{n-1}}\right\}\right)-\operatorname{Area}\left(\left\{\widehat{a}, \overline{P_{n-1} Z_{n-1}}\right\}\right)$.

Thus,

$$
\begin{aligned}
\operatorname{Area}\left(\left\{\widehat{a}, \widehat{P_{n-1} Z_{n-1}}\right\}\right) & =\left|\widetilde{P_{n-1} Z_{n-1}}\right|^{2} \frac{f(\gamma)-f(\gamma-2 \alpha)}{4} \\
& =\left|\overline{P_{n-1} Q_{n-1}}\right|^{2} \frac{2 \alpha f^{\prime}(\xi)}{16 \cos ^{2}(\gamma / 2)} \quad(\gamma-2 \alpha \leq \xi \leq \gamma) \\
& \geq\left|\overline{P_{n-1} Q_{n-1}}\right|^{2} \frac{\alpha f^{\prime}(0)}{8} \\
& =\left|\overline{P_{n-1} Q_{n-1}}\right|^{2} \frac{\alpha}{24} .
\end{aligned}
$$

The equality in (3) follows from the Mean Value Theorem, which states that $f(\gamma)-$ $f(\gamma-2 \alpha)=f^{\prime}(\xi) 2 \alpha$ where $\gamma-2 \alpha \leq \xi \leq \gamma$. Since $f^{\prime}(\theta)$ is monotonically increasing when $0<\theta<\pi$, and $2 \alpha<\gamma<\pi$, (4) follows as well.

It is easy to verify that

$$
\operatorname{Area}\left(\left\{\widehat{P_{n-1} Q_{n-1}}, \overline{P_{n-1} \overline{Q_{n-1}}}\right\}\right)=\left|\overline{P_{n-1} Q_{n-1}}\right|^{2} \frac{f(2 \gamma)}{4}
$$

and $f(2 \gamma) \cos ^{2}(\gamma / 2) \leq(2 \pi+1) / 8 \sin ^{2} \alpha$ when $2 \alpha<\gamma<\pi$. From (3) we have

$$
\begin{aligned}
\operatorname{Area}\left(\left\{\hat{a}, \widehat{P_{n-1} Z_{n-1}}\right\}\right) & =\operatorname{Area}\left(\left\{\widehat{P_{n-1} Q_{n-1}}\right\}\right) \frac{\alpha f^{\prime}(\xi)}{2 \cos ^{2}(\gamma / 2) f(2 \gamma)} \\
& \geq \operatorname{Area}\left(\left\{\widehat{P_{n-1} Q_{n-1}}\right\}\right) \frac{8 \alpha \sin ^{2}(\alpha)}{6(2 \pi+1)} .
\end{aligned}
$$


When $\widehat{P_{n-1} Q_{n-1}}$ is concave, $\widehat{a}$ must be concave. Using a similar argument as above, we can show that there exist constants $r_{\alpha}$ and $r_{\alpha}^{\prime}$ such that $\operatorname{Area}\left(\left\{\widehat{a}, \widehat{P_{n-1} Z_{n-1}}\right\}\right) \geq$ $r_{\alpha}\left|\widetilde{P_{n-1} Q_{n-1}}\right|^{2}$ and that $\operatorname{Area}\left(\left\{\widehat{a}, \widehat{P_{n-1} Z_{n-1}}\right\}\right) \geq r_{\alpha}^{\prime} \operatorname{Area}\left(\left\{\widehat{P_{n-1} Q_{n-1}}\right\}\right)$. From (1) and (5) and (2) and (6), then we have

$$
\begin{aligned}
& k_{\alpha}=\min \left\{\frac{f(\alpha)}{16}, \frac{\alpha}{24}, r_{\alpha}\right\}, \\
& k_{\alpha}^{\prime}=\min \left\{\frac{f(\alpha)}{4 f(4 \pi / 3)}, \frac{8 \alpha \sin ^{2} \alpha}{6(2 \pi+1)}, r_{\alpha}^{\prime}\right\} .
\end{aligned}
$$

Since $\alpha>0, f(\alpha)>0$ and $\sin ^{2} \alpha>0$. Thus, $k_{\alpha}$ and $k_{\alpha}^{\prime}$ are positive numbers.

We emphasize that the constants $k_{\alpha}$ and $k_{\alpha}^{\prime}$ are not dependent on $\gamma$ and hence the result can be extended to the other arcs, $\widehat{Q_{n-1} R_{n-1}}$ and $\widehat{R_{n-1} P_{n-1}}$. We are now ready for the main result of this section.

Theorem 2. Any planar, circular-arc drawing of $H_{n}$ that has constant angular resolution $\alpha>0$ has area $\Omega\left(c_{\alpha}^{n}\right)$ where $c_{\alpha}>1$.

Proof. Let $\Gamma_{n}^{*}$ be a planar, circular-arc drawing of $H_{n}$ with minimum area $A_{n}$. Let $B_{n-1}$ denote the area occupied by $H_{n-1}$ in $\Gamma_{n}^{*}$. Clearly, $B_{n-1} \geq A_{n-1}$. Then

$$
\begin{aligned}
& A_{n} \geq B_{n-1}+\operatorname{Area}\left(\mathcal{S}_{1}\right)+\operatorname{Area}\left(\mathcal{S}_{2}\right)+\operatorname{Area}\left(\mathcal{S}_{3}\right) \\
& \geq B_{n-1}+\frac{1}{2}\left[k_{\alpha}\left|\overline{P_{n-1} Q_{n-1}}\right|^{2}+k_{\alpha}^{\prime} \text { Area }\left(\left\{\widehat{P_{n-1} Q_{n-1}}, \overline{P_{n-1} Q_{n-1}}\right\}\right)\right. \\
& +k_{\alpha}\left|\overline{Q_{n-1} R_{n-1}}\right|^{2}+k_{\alpha}^{\prime} \operatorname{Area}\left(\left\{\widehat{Q_{n-1} R_{n-1}}, \overline{Q_{n-1} R_{n-1}}\right\}\right) \\
& \left.+k_{\alpha}\left|\overline{R_{n-1} P_{n-1}}\right|^{2}+k_{\alpha}^{\prime} \operatorname{Area}\left(\left\{\widehat{R_{n-1} P_{n-1}}, \overline{R_{n-1} P_{n-1}}\right\}\right)\right] \\
& \geq B_{n-1}+\frac{\min \left(k_{\alpha}, k_{\alpha}^{\prime}\right)}{2}\left[\left|\overline{P_{n-1} Q_{n-1}}\right|^{2}+\left|\overline{Q_{n-1} R_{n-1}}\right|^{2}+\left|\overline{R_{n-1} P_{n-1}}\right|^{2}\right. \\
& +\operatorname{Area}\left(\left\{\widehat{P_{n-1} Q_{n-1}}, \overline{P_{n-1} Q_{n-1}}\right\}\right) \\
& +\operatorname{Area}\left(\left\{\widehat{Q_{n-1} R_{n-1}}, \overline{Q_{n-1} R_{n-1}}\right\}\right) \\
& \left.+\operatorname{Area}\left(\left\{\widehat{R_{n-1} P_{n-1}}, \overline{R_{n-1} P_{n-1}}\right\}\right)\right] \\
& \geq B_{n-1}+\frac{\min \left(k_{\alpha}, k_{\alpha}^{\prime}\right)}{2}\left[\operatorname{Area}\left(\left\{\overline{P_{n-1} Q_{n-1}}, \overline{Q_{n-1} R_{n-1}}, \overline{R_{n-1} P_{n-1}}\right\}\right)\right. \\
& +\operatorname{Area}\left(\left\{\widehat{P_{n-1} Q_{n-1}}, \overline{P_{n-1} Q_{n-1}}\right\}\right) \\
& +\operatorname{Area}\left(\left\{\widehat{Q_{n-1} R_{n-1}}, \overline{Q_{n-1} R_{n-1}}\right\}\right) \\
& \left.+\operatorname{Area}\left(\left\{\widehat{R_{n-1} P_{n-1}}, \overline{R_{n-1} P_{n-1}}\right\}\right)\right] \\
& \geq B_{n-1}+\frac{\min \left(k_{\alpha}, k_{\alpha}^{\prime}\right)}{2} B_{n-1} \\
& \geq\left(1+\frac{\min \left(k_{\alpha}, k_{\alpha}^{\prime}\right)}{2}\right) A_{n-1} .
\end{aligned}
$$

Here (7) follows from Lemmas 4 and 5, while (8) follows from the fact that $B_{n-1} \geq \operatorname{Area}\left(\left\{\overline{P_{n-1} Q_{n-1}}, \overline{Q_{n-1} R_{n-1}}, \overline{R_{n-1} P_{n-1}}\right\}\right)+\operatorname{Area}\left(\left\{P_{n-1} Q_{n-1}, \overline{P_{n-1} Q_{n-1}}\right\}\right)+$ 
$\operatorname{Area}\left(\left\{\widehat{Q_{n-1} R_{n-1}}, \overline{Q_{n-1} R_{n-1}}\right\}\right)+\operatorname{Area}\left(\left\{\widehat{R_{n-1} P_{n-1}}, \overline{R_{n-1} P_{n-1}}\right\}\right)$. Let $c_{\alpha}=1+$ $\min \left(k_{\alpha}, k_{\alpha}^{\prime}\right)$.

Since $A_{1}$ is at least some constant $a_{1}>0$, by induction, $A_{n} \geq c_{\alpha}^{n-1} a_{1}$.

\section{Conclusion and Open Problems}

In this paper we prove that drawing a planar graph with good angular resolution using one circular arc per edge requires exponential area. We then show how to draw planar graphs with good angular resolution using polylines with at most one bend per edge on a grid of size $30 n \times 15 n$. Reducing the constants seems possible but this problem has not been explored yet.

The algorithm for drawing with one bend per edge immediately implies that we can draw planar graphs using two circular arcs with $C^{0}$-continuity, or using three circular arcs with $C^{1}$-continuity. However, it still remains to show whether we can draw using two circular arcs, or possibly two other degree- 2 curves, with $C^{l}$-continuity.

\section{Acknowledgments}

We thank Anhua Lin for helpful discussions and suggestions.

\section{References}

1. G. Di Battista, P. Eades, R. Tamassia, and I. Tollis. Graph Drawing: Algorithms for the Visualization of Graphs. Prentice-Hall, Englewood Cliffs, NJ, 1999.

2. M. Chrobak and T. Payne. A linear-time algorithm for drawing planar graphs. Information Processing Letters, 54:241-246, 1995.

3. H. de Fraysseix, J. Pach, and R. Pollack. How to draw a planar graph on a grid. Combinatorica, 10(1):4151, 1990.

4. I. Fáry. On straight lines representation of planar graphs. Acta Scientiarum Mathematicarum, 11:229-233, 1948.

5. M. Formann, T. Hagerup, J. Haralambides, M. Kaufmann, F. T. Leighton, A. Simvonis, E. Welzl, and G. Woeginger. Drawing graphs in the plane with high resolution. SIAM Journal of Computing, 22:1035$1052,1993$.

6. A. Garg and R. Tamassia. Planar drawings and angular resolution: algorithms and bounds. In Proceedings of the 2nd Annual European Symposium on Algorithms, pages 12-23, 1994.

7. M. T. Goodrich and C. G. Wagner. A framework for drawing planar graphs with curves and polylines. In Proceedings of the 6th Annual Symposium on Graph Drawing, pages 153-166, 1998.

8. C. Gutwenger and P. Mutzel. Planar polyline drawings with good angular resolution. In Proceedings of the 6th Annual Symposium on Graph Drawing, pages 167-182, 1998.

9. G. Kant. Drawing planar graphs using the Imc-ordering. In Proceedings of the 33rd Annual IEEE Symposium on Foundations of Computer Science, pages 101-110, 1992.

10. G. Kant. Algorithms for Drawing Planar Graphs. Ph.D. thesis, Department of Computer Science, University of Utrecht, Utrecht, 1993.

11. G. Kant. Drawing planar graphs using the canonical ordering. Algorithmica, 16:4-32, 1996 (special issue on Graph Drawing, edited by G. Di Battista and R. Tamassia).

12. S. Malitz and A. Papakostas. On the angular resolution of planar graphs. SIAM Journal of Discrete Mathematics, 7:172-183, 1994. 
13. W. Schnyder. Embedding planar graphs on the grid. In Proceedings of the 1 st ACM-SIAM Symposium on Discrete Algorithms (SODA), pages 138-148, 1990.

14. W. T. Tutte. How to draw a graph. Proceedings of the London Mathematical Society, 13(52):743-768, 1963.

15. K. Wagner. Bemerkungen zum vierfarbenproblem. Jahresbericht der Deutschen Mathematiker-Vereinigung, 46:26-32, 1936.

Received September 30, 1999, and in revised form March 27, 2000. Online publication October 25, 2000. 